\title{
Management of insomnia in sleep disordered breathing
}

\author{
Hennie C.J.P. Janssen ${ }^{1}$, Lisette N. Venekamp ${ }^{1}$, Geert A.M. Peeters ${ }^{1}$, \\ Angelique Pijpers ${ }^{1}$ and Dirk A.A. Pevernagie ${ }^{1,2}$
}

Affiliations: ${ }^{1}$ Sleep Medicine Center Kempenhaeghe, Heeze, The Netherlands. ${ }^{2}$ Dept of Internal Medicine and Paediatrics, Faculty of Medicine and Health Sciences, Ghent University, Ghent, Belgium.

Correspondence: D.A.A. Pevernagie, Dept of Internal Medicine and Paediatrics, Ghent University, Corneel Heymanslaan 10, Ghent, 9000, Belgium. E-mail: pevernagiedakempenhaeghe.nl

@ERSpublications

Obstructive sleep apnoea and insomnia frequently co-occur. Their pathophysiological relationship is intricate, and the resulting clinical condition may be difficult to treat. Insomnia treatment can be started first to alleviate symptoms of disturbed sleep. http://bit.ly/2kpwtEl

Cite this article as: Janssen HCJP, Venekamp LN, Peeters GAM, et al. Management of insomnia in sleep disordered breathing. Eur Respir Rev 2019; 28: 190080 [https://doi.org/10.1183/16000617.0080-2019].

ABSTRACT Both obstructive sleep apnoea (OSA) and chronic insomnia disorder are highly prevalent in the general population. Whilst both disorders may occur together by mere coincidence, it appears that they share clinical features and that they may aggravate each other as a result of reciprocally adverse pathogenetic mechanisms. Comorbidity between chronic insomnia disorder and OSA is a clinically relevant condition that may confront practitioners with serious diagnostic and therapeutic challenges. Current data, while still scarce, advocate an integrated and multidisciplinary approach that seems superior over the isolated treatment of each sleep disorder alone.

\section{Introduction}

Insomnia and sleep disordered breathing (SDB) are the two most frequent sleep disorders in the general population. Because of their high prevalence, there is a substantial chance of coincidence. When they occur together, implications for therapeutic management may arise. It is not clear whether these disorders should be treated separately according to prevailing standards, or whether a different, more integrated, approach should be preferred. While insomnia and SDB are classified as entities with different pathophysiologic mechanisms and clinical presentations, they share common features. This overlap may blur the nosological distinction between the two conditions and may confound the logical principles of their treatment. In this review, we expand on the semantic definitions of insomnia and SDB. We examine their co-occurrence, potential reciprocal influence and provide clues for treatment of the combined clinical condition.

\section{Definitions}

The fundament of insomnia is a predominant complaint of dissatisfaction with either duration or quality of sleep in combination with daytime impairments. Operational descriptions for this clinical picture have been provided by different scientific organisations and have gradually been adapted over time. The International Classification of Sleep Disorders (ICSD) published by the American Academy of Sleep Medicine (AASM) is frequently cited in the literature. The latest edition (ICSD-3) differs from the previous ones in that the discrimination between various subtypes of insomnia has been abandoned and "insomnia" is being defined as a disorder in its own right, irrespective of cause or comorbidities [1].

Provenance: Commissioned article, peer reviewed.

Received: 21 July 2019 | Accepted after revision: 05 Sept 2019

Copyright $\odot$ ERS 2019. This article is open access and distributed under the terms of the Creative Commons Attribution Non-Commercial Licence 4.0. 
Furthermore, a distinction is being made between chronic insomnia disorder, short-term insomnia disorder and other insomnia disorders. The scope of this review is limited to the discussion of chronic insomnia disorder, which is basically defined as a complaint of persistent sleep difficulty that is present despite adequate opportunities for sleep and that results in impaired daytime functioning [1]. Chronicity is denoted by the presence of symptoms occurring at least three times per week and persisting for at least 3 months. According to ICSD-3, the sleep/wake difficulty in insomnia should not be better explained by another sleep disorder. It should be kept in mind that various definitions of insomnia have been applied in the publications cited in this article. The lack of standardisation in this domain hampers the comparison of research results.

SDB is a collective name for obstructive sleep apnoea (OSA), central sleep apnoea and sleep-related hypoventilation syndromes. The present review deals mainly with OSA. The definition of this disorder has also been subject to substantial changes over time, favouring the apnoea/hypopnoea index (AHI) as the primary predictor or exposure variable for the clinical relevance of the disorder. According to the AASM, as published in the ICSD-3, OSA is characterised by three main diagnostic features [2]: 1) the presence of daytime and/or nocturnal symptoms including insomnia; 2) the presence of five or more predominantly obstructive events per hour; or 3) the presence of 15 or more predominantly obstructive events per hour. The ICSD-3 stipulates that 1 plus 2, or 3 alone, satisfy the diagnostic criteria of OSA. In contrast with insomnia, it is not required to exclude other sleep disorders that would better explain the clinical picture.

This would imply that whenever five or more predominantly obstructive events per hour are demonstrated in a sleep recording, symptoms of sleeplessness should not be classified as chronic insomnia disorder, but rather as OSA. Such classification, however, would be very impractical for daily routine care, because demographic research has shown that the prevalence of an elevated AHI may be extremely high in the general population [3,4]. All too often, genuine chronic insomnia disorder would be misclassified as OSA when an increased AHI would be taken as the prevailing diagnostic criterion. In the past decade, the usefulness of the AHI in establishing the diagnosis of clinically relevant OSA has been questioned. Scepticism has been fed by the absence of a clear relationship between the AHI and symptom severity [5-7]. Furthermore, recent therapeutic trials with positive airway pressure (PAP) failed to show a robust impact on relevant cardiovascular comorbidity in OSA patients who were enrolled based on AHI criteria $[8,9]$. These observations prompt for clinical characterisation of OSA on other features than the mere value of the AHI.

\section{Co-occurrence of insomnia and OSA}

Several publications have addressed the co-occurrence of chronic insomnia disorder and OSA. In a recent systematic review and meta-analysis, ZHANG et al. [10] have evaluated published data on the co-occurrence prevalence of insomnia and insomnia symptoms with OSA. A total of 37 studies were included in their analysis. In OSA patients, high rates were found for overall prevalence of insomnia (38\%), any insomnia complaints (36\%), difficulty falling asleep (18\%), difficulty maintaining sleep (42\%) and early morning awakenings (21\%). They also analysed the pooled prevalence rates of OSA based on different AHI criteria

TABLE 1 Interrelation between symptoms and clinical features associated with obstructive sleep apnoea (OSA) and insomnia

OSA Both Insomnia

Snoring

Breathing pauses

Breath holding, gasping, choking

Frequent arousals due to sleep disordered breathing events

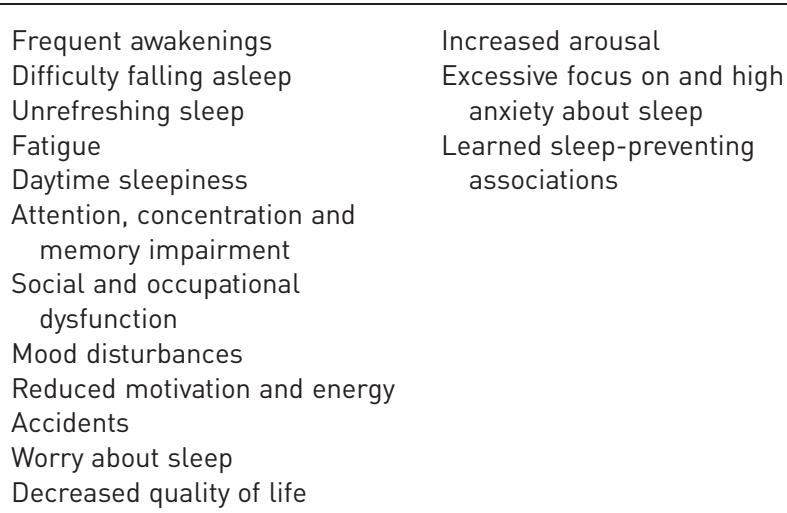

Frequent awakenings

Difficulty falling asleep

Unrefreshing sleep

Fatigue

Daytime sleepiness

Attention, concentration and memory impairment

Social and occupational dysfunction

Mood disturbances

Reduced motivation and energy

Accidents

Worry about sleep

Decreased quality of life

Increased arousal

Excessive focus on and high anxiety about sleep

Learned sleep-preventing associations

Reproduced from [11] with permission. 
in patients with insomnia. The rates were $35 \%$ (AHI $\geqslant 5$ events $\cdot h^{-1}$ ) and $29 \%$ (AHI $\geqslant 15$ events $\cdot h^{-1}$ ), respectively. These findings would suggest that OSA and insomnia mutually overlap in approximately a third of cases.

OSA and insomnia are dissimilar from a clinical and pathophysiological point of view. From a clinical perspective, these disorders can be modelled as orthogonal conditions, because insomnia is associated with increased arousal and alertness, whereas in OSA excessive daytime sleepiness is the most characteristic feature. Nevertheless, there is a substantial similarity between these two conditions regarding the nature of clinical manifestations. In a review of seven relevant studies, LuYsTER et al. [11] have reported on symptoms of OSA and insomnia, based on the ICSD. They found that only a minority of clinical characteristics are distinct, whereas most daytime and night-time complaints are shared by both disorders (table 1). They concluded that because of the substantial overlap in symptoms between insomnia and OSA the evaluation and treatment of either condition may be compromised and that multidisciplinary collaboration among sleep specialists is necessary. In this early review it was suggested that combination therapy, including both cognitive behavioural therapy (CBT) for insomnia and OSA treatment, resulted in greater improvements than either treatment strategy alone.

\section{Insomnia profile in OSA cluster analysis}

Recent research has focused on symptom scores and comorbidities of OSA to explore whether phenotypical subtypes exist among patients with this disorder. In an Icelandic sample of 822 newly diagnosed OSA patients with an AHI $\geqslant 15$ events $\mathrm{h}^{-1}$, three distinct clinical clusters were identified among which a disturbed sleep group represented $32.7 \%$ of the total cohort [12]. This cluster did not differ from the other two clusters (i.e. the minimally symptomatic group and the group with excessive daytime sleepiness) with respect to sex, body mass index or AHI. In a large international cohort of OSA patients, the existence of these clusters was confirmed and complemented with two other clusters. The disturbed sleep cluster comprised $19 \%$ of patients, with the highest rates of difficulty falling asleep (82.4\%), difficulty maintaining sleep (97.2\%), waking too early (77.3\%) and restless sleep (79.7\%). This cluster also had the highest rates of restless leg syndrome, hypertension, diabetes and cardiovascular disease compared to the other clusters (minimal symptoms, upper airway symptoms with sleepiness, upper airway symptoms dominant and sleepiness dominant) [13]. The higher frequency of essential hypertension and cerebrovascular disease in OSA patients with insomnia complaints compared to OSA patients without insomnia complaints was also found in a large retrospective study [14].

These cluster analyses evoke the existence of distinct clinical phenotypes among the large and ever expanding OSA patient population. One of these phenotypes is clearly disturbed sleep showing characteristics of chronic insomnia disorder. It remains to be determined if this trait represents a treatable characteristic of OSA or signifies an unrelated condition that would require non-respiratory therapeutic interventions.

\section{The relationship between OSA and insomnia}

It proves difficult to tease out causal relationships between OSA and insomnia in individual patients. Both conditions may be unrelated, could be a clinical trait of one another, or could even negatively affect each other. Often, empirical treatment is needed to clarify causality, as differential therapeutic responses may point out whether the OSA condition is primary or subordinate to the insomnia component and vice versa.

\section{OSA as a cause of insomnia}

Repeated obstructive respiratory events may be instrumental to the development of sleeplessness in some OSA patients. Several mechanisms are believed to play a role [15]. Apnoeas are invariably associated with cortical arousals from sleep. These short interruptions of sleep may be perceived as awakenings or continued wakefulness if they are vigorous and occur repeatedly. Nocturia is a frequent complication of OSA. Patients have to get out of bed several times, and this mere fact is an obvious cause of sleep disruption. Some patients expand the time in bed to compensate for not being refreshed upon awakening. They may take periods of rest during the daytime and increase caffeine intake. These behavioural patterns are also typical of psychophysiological insomnia, meaning that measures to alleviate daytime somnolence may be conducive to developing problems with initiation and maintenance of sleep. Finally, apnoea-induced arousals that are associated with sensations of breathlessness may promote anxiety, which in turn may prime cognitive processes towards increased alertness.

If disturbed breathing is indeed the cause of insomnia, OSA targeted treatment will improve insomnia complaints. Restoration of sleep initiation and continuity under PAP treatment has been shown in several studies. Glidewell et al. [16] studied 68 OSA patients, 53 of whom had pre-treatment insomnia symptoms. In this group, PAP therapy reduced insomnia severity scores from moderate/severe to no/mild symptoms 
in $45 \%$ (24 out of 53 subjects). Nevertheless, more than half of the patients had persistent insomnia that was not responsive to PAP therapy. In another trial, the effect of PAP treatment was evaluated in a large OSA cohort that was stratified according to phenotype [17]. In the disturbed sleep group, PAP users and non-users generally reported similar changes in insomnia symptoms after 2 years, indicating that factors other than PAP treatment effects may play a role in the modulation of insomnia complaints. Furthermore, differences in the type of insomnia seemed to influence the results. The disturbed sleep characteristic was further subdivided into difficulty falling asleep (initial insomnia), waking up often during the night (middle insomnia) and waking up early in the morning being unable to fall back asleep (late insomnia). Improvement of middle insomnia at 2 years follow-up of PAP treatment was much more likely to occur among patients who were adherent to PAP treatment as compared to noncompliant individuals. In contrast, symptoms of initial and late insomnia were more likely to persist and seemed resistant to PAP use. In another study, it was shown that PAP treatment may worsen insomnia, particularly in the initial and late insomnia subgroups [18]. In conclusion, symptoms of insomnia may be PAP responsive in certain patients. However, this effect is hard to predict and remains a post hoc finding in patients who are sufficiently compliant to PAP treatment.

\section{Insomnia as a cause of OSA}

In addition to the well-known anatomical predisposition to OSA, non-anatomical factors play a substantial role in the pathophysiology of OSA in more than half of the subjects [19] and the relative contribution of each of them can vary markedly among OSA patients [20]. These non-anatomical features include low pharyngeal muscle responsiveness, oversensitive ventilatory control and a low respiratory arousal threshold during sleep. The latter trait was found to be prominent in about $37 \%$ of the cases. Patients with a low arousal threshold awaken prematurely in response to the increased respiratory resistance that occurs during upper airway narrowing. This pathophysiological feature may act in two ways, either as a mechanism causing insomnia in OSA (as described above), but also as a condition that predisposes to SDB in insomnia. Frequent awakenings and subsequent transitions to sleep invariably invoke withdrawal of the wakefulness stimulus, which causes breathing instability and predisposes to upper airway obstruction. This effect may be greater in OSA patients than in healthy subjects [21]. Circumstantial evidence from clinical practice supports this interaction (personal observations). Not infrequently, a temporary increase in AHI can be observed on the readout of PAP devices in OSA patients who are otherwise well-controlled with PAP therapy, but who suffer from transient bouts of insomnia (figure 1). Moreover, treatment with hypnotics has been shown to reduce OSA severity in OSA patient subgroups with a low respiratory arousal threshold. ECKERT and co-workers [22, 23] have successfully used eszopiclone and trazodone in OSA patients to increase the arousal threshold and to lower the AHI. These effects, being modest on average, confirm the multifactorial nature of OSA. It is intriguing to hypothesise that factors such as high loop gain and low arousal threshold might be related to clinical conditions such as stress and anxiety, which are often implicated in the perpetuation of chronic insomnia disorder. This hypothesis, however, remains to be proven.

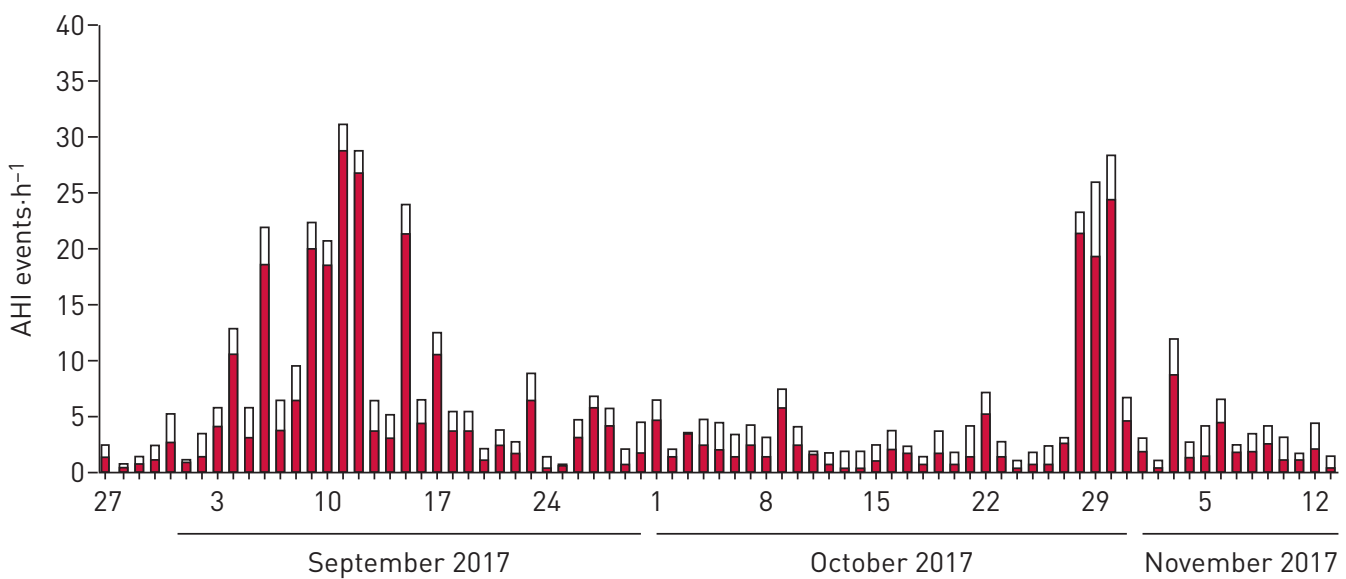

FIGURE 1 Temporary insomnia-induced increases of apnoea-hypopnea index (AHI) under continuous positive airway pressure (CPAP) therapy. In a 75-year-old male patient diagnosed with obstructive sleep apnoea (AHI 65 events $\cdot h^{-1}$ J, CPAP administered at a level of $8 \mathrm{cmH}_{2} \mathrm{O}$ was effective at controlling the AHI. He experienced two episodes of insomnia as a result of temporary marital disharmony. During these incidents AHI increased dramatically, although CPAP settings and use of drugs or alcohol remained unchanged. When normal sleep resumed the $\mathrm{AHI}$ fell back to baseline levels. 
Is there a reciprocal influence between insomnia and sleep apnoea?

A mutual reinforcement of negative effects ("positive feedback loop") may occur when OSA and insomnia are both present (figure 2). In this instance, the above-mentioned causal relationships are bidirectional in nature. The acronym "COMISA" has been coined by some authors to denote the intricate connection between both pathological entities and to point out that the clinical presentation of the comorbid condition may actually be worse than expected from the single contribution of each individual component $[14,15,24]$. In line with this hypothesis stands the observation that some patients with COMISA experience more severe sleep disturbance than patients with either disorder alone [25]. In COMISA patients, daytime symptom scores tend to be higher and rates of psychiatric and cardiovascular comorbidities are reportedly more frequent [15].

Another common finding is that COMISA is more difficult to treat than either OSA or insomnia alone. Reduced compliance to PAP therapy is frequently observed. EYsTeINSDOTTiR et al. [26] followed 796 OSA patients (AHI $\geqslant 15$ events $\cdot \mathrm{h}^{-1}$ ) after commencement of PAP treatment over a mean period of 7 years. It was shown that having initial or late insomnia complaints at the start was associated with quitting PAP treatment early among non-obese patients. Similar findings came out of a French cohort of OSA patients with a mean initial AHI of $34.6 \pm 20.7$ events $\cdot h^{-1}$ [27]. It appeared that sleep onset and sleep maintenance insomnia were inversely related to PAP compliance. Inducing or worsening insomnia complaints is a frequent cause of noncompliance to PAP therapy [11]. Similarly, treatment of insomnia with hypnotics may worsen SDB. In a recent retrospective case-control study, evidence was found that the use of benzodiazepines might increase the risk of acute respiratory failure in OSA patients [28].

Eventually, one should realise that, given the high prevalence of both insomnia and OSA, the co-occurrence of both conditions can be due to mere coincidence in any given patient. On the one hand, both may be significant to the constellation of complaints (and thus warrant treatment), and on the other hand, either one could be of less importance, or even be irrelevant. Reduced total sleep time or sleep efficiency in the absence of daytime symptoms should not be labelled as insomnia [1]. An increased AHI, even in the presence of daytime symptoms, does not necessarily qualify as clinically relevant OSA [29]. Proficient clinical judgement is paramount in the proper assessment of this complex problem.

\section{Methodological issues}

Appraisal of the literature on the co-existence of insomnia and OSA confronts the reader with several methodological issues. The formulation of a working hypothesis on the complex interaction between these two entities is likewise hampered by conceptual limitations. In recent systematic reviews, available studies were compared with respect to prevalence of co-occurrence of insomnia and OSA [10] and treatment of comorbid insomnia and OSA [24]. Both studies draw attention to the limited number of high-quality publications and to the inconsistency of the methodology. Demographic inclusion criteria vary highly among studies. Different definitions and cut-off values of markers for clinical severity are used for both

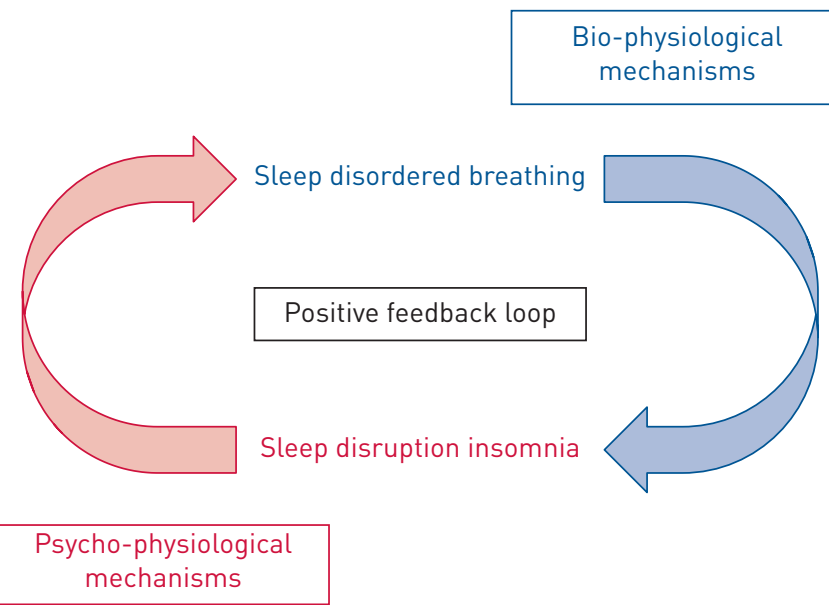

FIGURE 2 Potential pathophysiological feedback mechanism of COMISA. This scheme represents the potential positive feedback loop between sleep disordered breathing (obstructive sleep apnoeal and sleep disruption (insomnia), explaining why the resultant clinical picture may be worse than what can be expected from the contribution of either component alone. Moreover, as a result of this vicious circle, interventions geared to treat either insomnia or obstructive sleep apnoea may have adverse effects to the comorbid component le.g. sleep restriction therapy may aggravate somnolence in obstructive sleep apnoea and continuous positive airway pressure therapy may worsen insomnia). 
insomnia and OSA. Definitions on adherence to treatment are also disparate. Moreover, studies are coloured by the different disciplines, where pulmonologists and sleep physicians tend to put emphasis on apnoeic events, whereas psychologists focus more on insomnia features. One study concluded that the contemporary level of evidence is not yet sufficient for a meta-analysis on COMISA therapy [24]. It is a challenge for future research to standardise definitions and methods in order to come up with comparable research results.

\section{Clues for clinical management Assessment of combined insomnia with OSA}

If an evaluation of insomnia/OSA is warranted, an efficient diagnostic procedure is mandatory [30]. The diagnosis of insomnia is founded on comprehensive assessment of several clinical aspects and includes proficient history taking, the recording of sleep diaries and physical examination. Complementary techniques such as actigraphy may be indicated in certain cases [31].

Polysomnography, which is not routinely recommended for the assessment of insomnia [31], is carried out in patients with suspected OSA to quantify the obstructive breathing events. Polysomnography will clearly show the insomnia-related disturbances of sleep initiation and sleep maintenance in the hypnogram. Polygraphy does not include recording of neurophysiological variables and is intrinsically ill-suited to document the characteristics of insomnia. Moreover, the AHI will be underestimated with polygraphy because wakefulness and arousals go unnoticed.

\section{Empirical therapeutic approach}

Given the fact that medical evidence is actually lacking, no formal recommendations or guidelines on treatment of insomnia/OSA can be made. Treatment of this composite condition relies on clinical judgement and implies the mastering of pertinent knowledge and skills in the field of clinical sleep medicine by the practitioner. Sleep physicians and sleep psychologists should be aware of their mutual areas of interest. In light of this, the importance of an integrated, interdisciplinary approach to insomnia/ OSA must be emphasised.

Clinical judgement should guide whether to start with PAP therapy (or another suitable treatment option for OSA) or CBT for insomnia, or both. It has been demonstrated in a retrospective trial including 455 subjects that CBT for insomnia can effectively treat insomnia in patients with comorbid OSA [32]. Significant improvements were found in insomnia symptoms, global insomnia severity, and daytime functioning measures during and following treatment. Furthermore, improvements were not different between patients with or without comorbid OSA. Finally, it became evident that the effectiveness of CBT for insomnia was not impaired by the presence of OSA. The same group of investigators recently showed that in OSA patients with comorbid insomnia, CBT for insomnia prior to initiating continuous positive airway pressure (CPAP) treatment improves CPAP use and insomnia symptoms compared to commencing CPAP without CBT for insomnia [33]. While these findings would benefit from replication by other research groups, they deliver support to the contention that patients with comorbid insomnia and OSA can be safely and effectively treated with CBT for insomnia prior to or together with the start of OSA therapy.

\section{Conclusions}

Current literature indicates that OSA and insomnia frequently co-occur. The interaction between these two conditions may be variable and range from insignificant to mutually detrimental as a result of a positive feedback loop. Insight into these presumed interactions is hampered by semantic issues, such as the lack of universally accepted operational definitions of insomnia and OSA. The co-incidence of insomnia with OSA is a condition that is often puzzling to practitioners. Recent evidence suggests that CBT for insomnia may be started as first-line treatment for this comorbid disorder. Following alleviation of insomnia symptoms, treatment of OSA may ensue. The intricacies of combined OSA/insomnia warrant management by practitioners trained in clinical sleep medicine in the context of an integrated and multidisciplinary care setting.

Conflict of interest: None declared.

\section{References}

1 Chronic insomnia disorder. In: Sateia M, ed. The International Classification of Sleep Disorders. 3rd Edn. Darien, American Academy of Sleep Medicine, 2014; pp. 21-41.

2 Obstructive sleep apnea: adult. In: Sateia M, ed. The International Classification of Sleep Disorders. 3rd Edn. Darien, American Academy of Sleep Medicine, 2014; pp. 53-62.

3 Heinzer R, Vat S, Marques-Vidal P, et al. Prevalence of sleep-disordered breathing in the general population: the HypnoLaus study. Lancet Respir Med 2015; 3: 310-318.

4 Heinzer R, Marti-Soler H, Haba-Rubio J. Prevalence of sleep apnoea syndrome in the middle to old age general population. Lancet Respir Med 2016; 4: e5-e6. 
5 Kingshott RN, Engleman HM, Deary IJ, et al. Does arousal frequency predict daytime function? Eur Respir J 1998; 12: $1264-1270$.

6 Tkacova R, McNicholas WT, Javorsky M, et al. Nocturnal intermittent hypoxia predicts prevalent hypertension in the European Sleep Apnoea Database cohort study. Eur Respir J 2014; 44: 931-941.

7 Turnbull CD, Stradling JR. To screen or not to screen for obstructive sleep apnea, that is the question. Sleep Med Rev 2017; 36: 125-127.

8 McEvoy RD, Antic NA, Heeley E, et al. CPAP for prevention of cardiovascular events in obstructive sleep apnea. N Engl J Med 2016; 375: 919-931.

9 McNicholas WT, Bassetti CL, Ferini-Strambi L, et al. Challenges in obstructive sleep apnoea. Lancet Respir Med 2018; 6: 170-172.

10 Zhang Y, Ren R, Lei F, et al. Worldwide and regional prevalence rates of co-occurrence of insomnia and insomnia symptoms with obstructive sleep apnea: a systematic review and meta-analysis. Sleep Med Rev 2019; 45: 1-17.

11 Luyster FS, Buysse DJ, Strollo PJ Jr. Comorbid insomnia and obstructive sleep apnea: challenges for clinical practice and research. J Clin Sleep Med 2010; 6: 196-204.

12 Ye L, Pien GW, Ratcliffe SJ, et al. The different clinical faces of obstructive sleep apnoea: a cluster analysis. Eur Respir J 2014; 44: 1600-1607.

13 Keenan BT, Kim J, Singh B, et al. Recognizable clinical subtypes of obstructive sleep apnea across international sleep centers: a cluster analysis. Sleep 2018; 41: https://doi.org/10.1093/sleep/zsx214.

14 Gupta MA, Knapp K. Cardiovascular and psychiatric morbidity in obstructive sleep apnea (OSA) with insomnia (sleep apnea plus) versus obstructive sleep apnea without insomnia: a case-control study from a Nationally Representative US sample. PLoS One 2014; 9: e90021.

15 Sweetman AM, Lack LC, Catcheside PG, et al. Developing a successful treatment for co-morbid insomnia and sleep apnoea. Sleep Med Rev 2017; 33: 28-38.

16 Glidewell RN, Renn BN, Roby E, et al. Predictors and patterns of insomnia symptoms in OSA before and after PAP therapy. Sleep Med 2014; 15: 899-905.

17 Pien GW, Ye L, Keenan BT, et al. Changing faces of obstructive sleep apnea: treatment effects by cluster designation in the Icelandic Sleep Apnea Cohort. Sleep 2018; 41: https://doi.org/10.1093/sleep/zsx201.

18 Bjornsdottir E, Janson C, Sigurdsson JF, et al. Symptoms of insomnia among patients with obstructive sleep apnea before and after two years of positive airway pressure treatment. Sleep 2013; 36: 1901-1909.

19 Eckert DJ, White DP, Jordan AS, et al. Defining phenotypic causes of obstructive sleep apnea. Identification of novel therapeutic targets. Am J Respir Crit Care Med 2013; 188: 996-1004.

20 Eckert DJ, Wellman A. Physiological phenotypes. In: Barbé F, Pépin JL, eds. Obstructive Sleep Apnoea (ERS Monograph). Sheffield, European Respiratory Society, 2015; pp. 9-23.

21 Fogel RB, Trinder J, White DP, et al. The effect of sleep onset on upper airway muscle activity in patients with sleep apnoea versus controls. J Physiol 2005; 564: 549-562.

22 Eckert DJ, Owens RL, Kehlmann GB, et al. Eszopiclone increases the respiratory arousal threshold and lowers the apnoea/hypopnoea index in obstructive sleep apnoea patients with a low arousal threshold. Clin Sci (Lond) 2011; 120: 505-514.

23 Eckert DJ, Malhotra A, Wellman A, et al. Trazodone increases the respiratory arousal threshold in patients with obstructive sleep apnea and a low arousal threshold. Sleep 2014; 37: 811-819.

24 Bahr K, Camara RJA, Gouveris H, et al. Current treatment of comorbid insomnia and obstructive sleep apnea with CBTI and PAP-therapy: a systematic review. Front Neurol 2018; 9: 804.

25 Bianchi MT, Williams KL, McKinney S, et al. The subjective-objective mismatch in sleep perception among those with insomnia and sleep apnea. J Sleep Res 2013; 22: 557-568.

26 Eysteinsdottir B, Gislason T, Pack AI, et al. Insomnia complaints in lean patients with obstructive sleep apnea negatively affect positive airway pressure treatment adherence. J Sleep Res 2017; 26: 159-165.

27 Philip P, Bioulac S, Altena E, et al. Specific insomnia symptoms and self-efficacy explain CPAP compliance in a sample of OSAS patients. PLoS One 2018; 13: e0195343.

28 Wang SH, Chen WS, Tang SE, et al. Benzodiazepines associated with acute respiratory failure in patients with obstructive sleep apnea. Front Pharmacol 2018; 9: 1513.

29 Bixler EO, Vgontzas AN, Gaines J, et al. Moderate sleep apnoea: a "silent" disorder, or not a disorder at all? Eur Respir J 2016; 47: 23-26.

30 Pevernagie DA. Insomnia. In: Simonds AK, de Backer W, eds. ERS Handbook of Respiratory Sleep Medicine. Sheffield, European Respiratory Society, 2012; pp. 53-60.

31 Riemann D, Baglioni C, Bassetti C, et al. European guideline for the diagnosis and treatment of insomnia. J Sleep Res 2017; 26: 675-700.

32 Sweetman A, Lack L, Lambert S, et al. Does comorbid obstructive sleep apnea impair the effectiveness of cognitive and behavioral therapy for insomnia? Sleep Med 2017; 39: 38-46.

33 Sweetman A, Lack L, Catcheside PG, et al. Cognitive and behavioral therapy for insomnia increases the use of continuous positive airway pressure therapy in obstructive sleep apnea participants with co-morbid insomnia: A randomized clinical trial. Sleep 2019; https://doi.org/10.1093/sleep/zsz178. 